\title{
Acute stroke intervention after transcatheter aortic valve replacement
}

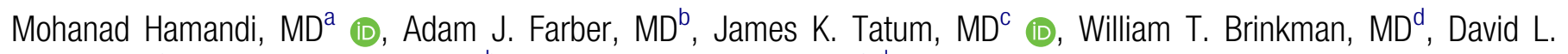 \\ Brown, $\mathrm{MD}^{\mathrm{e}}$, Mark E. Lawrence, $\mathrm{DO} \mathrm{b}^{\mathrm{b}}$, and Michael J. Mack, MD ${ }^{\mathrm{a}, \mathrm{d}}$ \\ ${ }^{a}$ Department of Cardiovascular Research, Baylor Scott \& White Research Institute at The Heart Hospital Plano, Plano, Texas; 'Department \\ of Interventional Cardiology, Baylor Scott \& White Medical Center, Temple, Texas; 'Department of Interventional Neuroradiology, Baylor \\ Scott \& White Medical Center, Plano, Texas; 'Department of Cardiothoracic Surgery, Baylor Scott \& White at The Heart Hospital Plano,

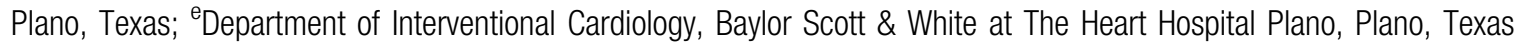

\section{ABSTRACT}

Stroke is a devastating complication in patients undergoing transcatheter aortic valve replacement (TAVR). We sought to determine whether early stroke intervention after TAVR would improve the neurological outcomes. Two patients experienced stroke immediately after TAVR, one treated with mechanical thrombectomy and localized Iytic therapy and one treated with systemic lytic therapy. Our limited experience showed that early stroke intervention with either treatment option may reduce the risk of complications and improve neurological outcomes.

KEYWORDS Lytic therapy; mechanical thrombectomy; stroke; transcatheter aortic valve replacement

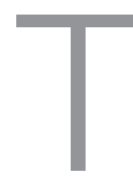

ranscatheter aortic valve replacement (TAVR) has emerged as a treatment choice for high-surgical-risk patients with aortic stenosis and as a treatment alternative for patients with intermediate surgical risk. The risk of stroke or transient ischemic attack is highest early after TAVR and is associated with increased morbidity and mortality. ${ }^{1,2}$ Recent reports from PARTNER (Placement of Aortic Transcatheter Valve) and CoreValve trials showed that high-risk patients experienced early stroke $3.3 \%$ to $4.1 \%$ of the time. ${ }^{1,3}$ The etiology of post-TAVR stroke is most commonly debris embolization during the procedure. ${ }^{4}$ The histopathologic analysis of the debris varies, though thrombus formation occurs in almost every case. ${ }^{5}$ Though stroke is a devastating complication of TAVR, there is little experience with early intervention to improve outcomes. In this report, we demonstrate the possible benefit of early post-TAVR stroke intervention with two different management strategies that led to marked improvement in the patients' neurological status.

\section{CASE 1}

A 73-year-old man presented with progressive fatigue, intermittent left-sided chest pain with occasional dizziness, and lower-extremity edema. His past medical history was significant for triple-vessel coronary artery bypass surgery, deep vein thrombosis, and pulmonary embolism, and he was diagnosed with heparin-induced thrombocytopenia. In addition, one of his vein grafts was positioned directly behind the sternum. There was no history of prior transient ischemia or stroke. Transthoracic echocardiogram revealed severe aortic stenosis with preserved left ventricular systolic function. The patient was offered TAVR. An Edwards Sapien S3 26-mm Bioprosthetic valve was implanted via the transfemoral route with moderate sedation. Bivalirudin was used throughout the procedure, and no complication was noted. Postprocedure, the patient had decreased responsiveness, dysarthria, and right-sided hemiparesis. Emergent brain computed tomographic angiography (CTA) revealed a filling defect at the left middle cerebral artery bifurcation (Figure 1). The patient

Corresponding author: Michael J. Mack, MD, Department of Cardiovascular Research, Baylor Scott \& White Research Institute, The Heart Hospital Baylor Plano, 4708 Alliance Blvd., Plano, TX 75093 (e-mail: Michael.Mack@bswhealth.org)

Color versions of one or more of the figures in this article can be found online at www.tandfonline.com/ubmc.

Received May 24, 2018; Revised July 8, 2018; Accepted July 9, 2018. 


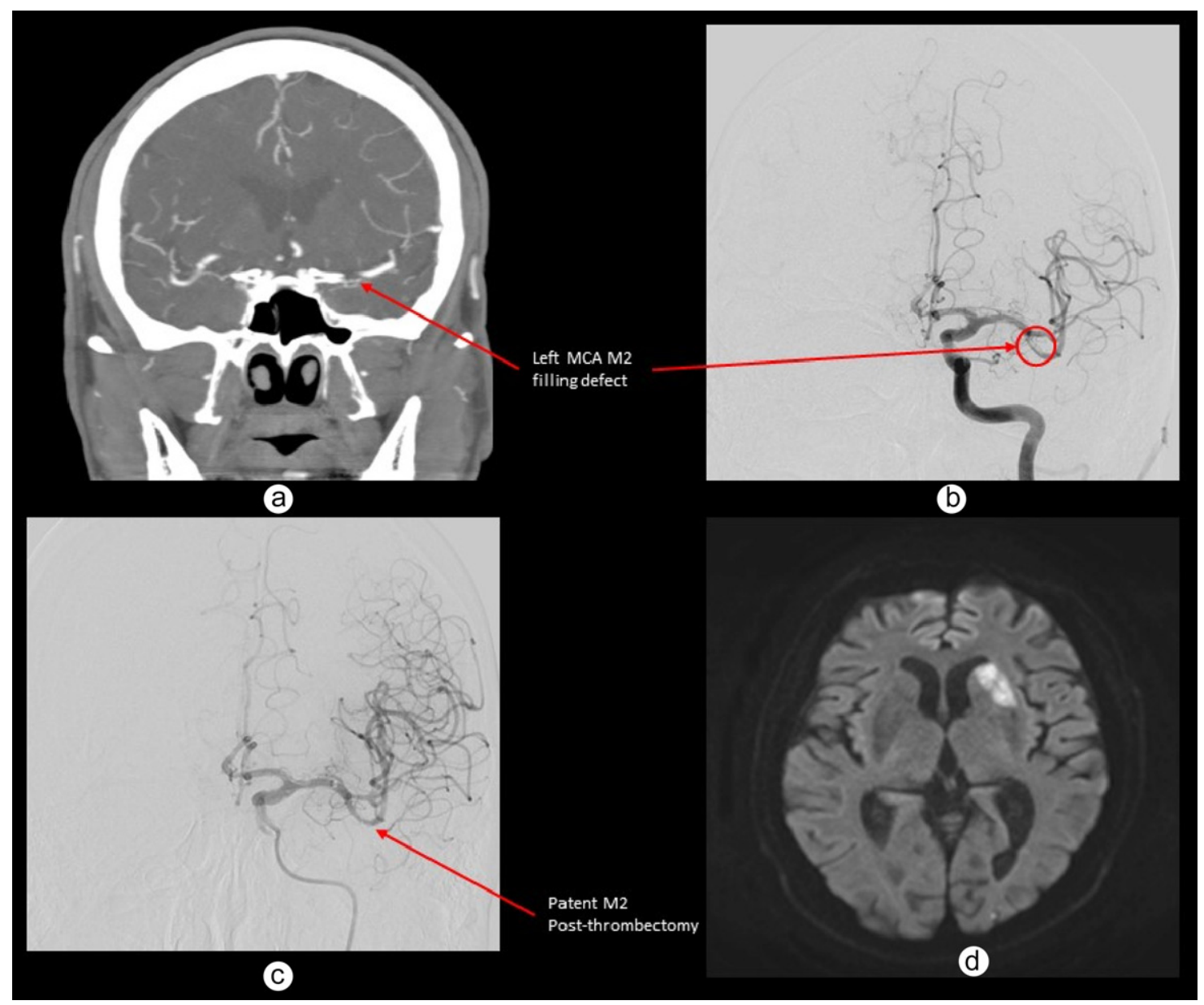

Figure 1. Case 1. (a) Computed tomography angiography (CTA) of the head, coronal view, reveals a left distal M1 segment/proximal M2 segment filling defect representing thrombus. (b, c) Anteroposterior angiogram with left internal carotid artery injection demonstrates (b) a filling defect in the inferior division of the left middle cerebral artery (MCA) proximal M2 segment reflecting thrombus and (c) Thrombolysis in Cerebral Infarction 3 recanalization of the left MCA following thrombectomy. (d) Diffusion-weighted MRI of the brain demonstrates increased signal in the left caudate head and anterior putamen reflecting restricted diffusion and acute infarct. A punctate embolic infarct is also noted in the left lateral occipital lobe.

underwent left middle cerebral artery mechanical thrombectomy and lysis. By the time of discharge 9 days later, the patient's neurological deficit totally resolved and he was neurologically intact with fluent speech. Transthoracic echocardiography revealed trivial perivalvular leak but otherwise normal function of the bioprosthetic valve.

\section{CASE 2}

An 81-year-old man underwent TAVR for symptomatic severe aortic stenosis. Past medical history included coronary artery disease, hypertension, hyperlipidemia, esophageal reflux, lower-extremity deep venous thrombosis, prostate cancer requiring radical prostatectomy, ataxia, and retropulsion thought to be due to cerebellar ataxia. There was no history of stroke or transient ischemic attack, and recent magnetic resonance imaging (MRI) was negative. Transthoracic echocardiography demonstrated severe aortic stenosis, severe left ventricular hypertrophy, and preserved systolic function. Fifteen days prior to the TAVR, a percutaneous coronary intervention with drug-eluting stents placed in the mid left anterior descending artery and right coronary artery was performed. Ticagrelor and aspirin were continued through the procedure.

Implantation of an Edwards Sapien S3 26-mm bioprosthetic valve was performed via the transfemoral route with moderate sedation and no complication noted during the procedure. No cerebral protection was utilized. Immediately following the procedure, neurologic function was normal. Thirty-eight minutes after deployment of the bioprosthetic valve in the postanesthesia care unit, the patient developed expressive aphasia, dysarthria, right facial droop, and right hemiparesis. The initial National Institutes of Health Stroke Scale score was 17. CT/CTA of the brain was performed 30 minutes after onset and demonstrated no emergent large vessel occlusion or other acute process. Postprocedure partial thromboplastin time was 34; hemodynamics remained stable, and neurologic deficits minimally changed, with a National Institutes of Health Stroke Scale score of 14. CTA showed left middle cerebral artery partial occlusion of M4 segment (Figure 2a). The stroke team administered a thrombolytic agent, alteplase (Activase, Genentech, South San Francisco, CA), at $0.9 \mathrm{mg} / \mathrm{kg}$ over an hour with $10 \%$ of the dose given as an initial bolus. Within 8 hours of administration, most of 


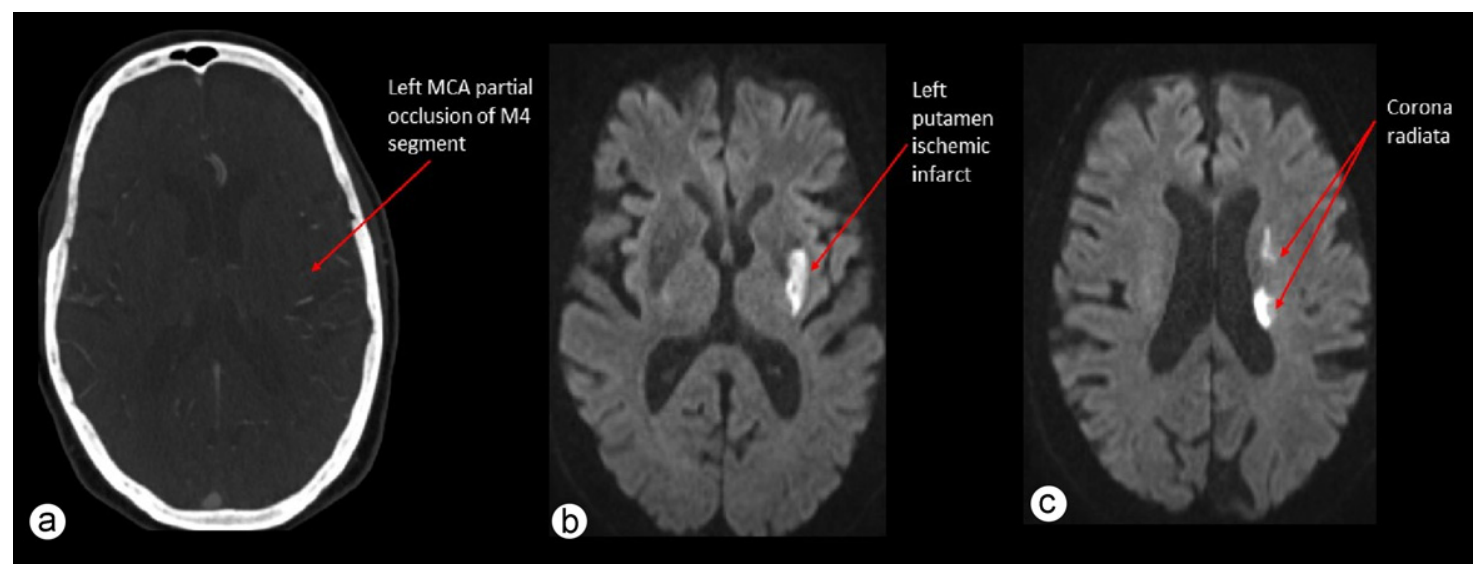

Figure 2. Case 2. (a) Axial computed tomography angiography reveals a filling defect in the M4 segment of the left middle cerebral artery. (b, c) Diffusionweighted MRI demonstrates increased signal in the left putamen and corona radiata consistent with acute ischemic infarcts.

his symptoms resolved. Twenty-four hours after alteplase administration, the patient had mild dysarthria and mild right arm weakness, with a National Institutes of Health Stroke Scale score of 2. Subsequent MRI of the brain demonstrated acute infarcts involving the left lentiform nucleus and left caudate body without intracranial hemorrhage (Figures 2b, 2c). Transthoracic echocardiography demonstrated mild periprosthetic leak but otherwise normal function of the prosthetic valve. The absence of a large-vessel occlusion on CTA imaging precluded mechanical thrombectomy or localized lytic therapy.

\section{DISCUSSION}

Stroke is a feared complication following TAVR and is associated with increased morbidity and mortality. Periprocedural and postprocedural stroke prevention and acute intervention are of highest importance for advancing TAVR for lower-risk patients. Mechanical thrombectomy and systemic thrombolysis, each used in one patient acutely after TAVR, appeared to be associated with significant improvement in the neurologic outcomes. Early risk of stroke suggests that embolic prevention devices and appropriate pharmacotherapy may significantly reduce the severity of the complication.

\section{ORCID}

Mohanad Hamandi (DD http://orcid.org/0000-0002-9746-5685

James K. Tatum (D) http://orcid.org/0000-0002-3217-2478

1. Kapadia S, Agarwal S, Miller DC, et al. Insights into timing, risk factors, and outcomes of stroke and transient ischemic attack after transcatheter aortic valve replacement in the PARTNER trial (Placement of Aortic Transcatheter Valves). Circ Cardiovasc Interv. 2016;9:e02981. doi:10.1161/CIRCINTERVENTIONS. 115.002981.

2. Stortecky S, Windecker S. Stroke: an infrequent but devastating complication in cardiovascular interventions. Circulation 2012;126:2921-2924. doi:10.1161/CIRCULATIONAHA.112.149492.

3. Kleiman NS, Maini BJ, Reardon MJ, et al. Neurological events following transcatheter aortic valve replacement and their predictors: a report from the CoreValve trials. Circ Cardiovasc Interv. 2016;9:e003551. doi:10.1161/CIRCINTERVENTIONS.115.003551.

4. Van Mieghem NM, Schipper ME, Ladich E, et al. Histopathology of embolic debris captured during transcatheter aortic valve replacement. Circulation 2013;127:2194-2201. doi:10.1161/CIRCULATIONAHA. 112.001091.

5. Kapadia SR, Kodali S, Makkar R, et al. Protection against cerebral embolism during transcatheter aortic valve replacement. J Am Coll Cardiol. 2017;69:367-377. doi:10.1016/j.jacc.2016.10.023. 